\title{
¿EXISTE UN PATRÓN DIFERENCIAL DE CONDUCTA DE ENFERMEDAD Y ADHESIÓN AL TRATAMIENTO DE LA INFECCIÓN POR VIH/SIDA EN PACIENTES CON HISTORIA DE ABUSO DE DROGAS?
}

\author{
RAFAEL BALLESTER, IDOIA REINOSO, ANA CAMPOS y SANTIAGO GARCÍA
}

\author{
Universidad Jaime I de Castellón
}

(Aceptado en febrero de 2003)

\begin{abstract}
En este artículo se analiza la posible existencia de un patrón diferencial de conducta de enfermedad y adhesión al tratamiento de la infección por VIH/SIDA en 69 pacientes con o sin historia de abuso de drogas (AD/NAD). Se evalúan variables directamente relacionadas con la adhesión y otras más generales de conducta de enfermedad de los pacientes. Los resultados muestran algunas diferencias estadísticamente significativas entre los pacientes AD y NAD en algunas variables de conducta de enfermedad. Asi, los pacientes $\mathrm{AD}$ han compartido en mayor medida jeringas, han padecido más ETS, su vía percibida de infección es la sanguínea, el motivo de realizarse las pruebas ha sido más la conciencia de haber realizado prácticas de riesgo, valoran la enfermedad como más grave, tienen peores expectativas acerca de la eficacia del tratamiento y peor relación con el facultativo. Además, la tendencia clínica observada es que los pacientes $\mathrm{AD}$ presentan un patrón más negativo de adhesión al tratamiento que los pacientes NAD, aunque las diferencias no alcanzan significación estadística.
\end{abstract}

Palabras clave: VIH, SIDA, adhesión al tratamiento, abuso de drogas

Is there a differential pattern of illness behaviour and adherence to treatment of HIV infection/AIDS in patients with substance abusing history?

In this article, we investigate the possible existence of a differential pattern of illness behaviour and adherence to treatment in 69 Spanish HIV/AIDS patients with or without substance abuse history (DA/NDA). Variables directly related to adherence to treatment and more general illness behaviour variables are evaluated. Results show some significant statistical differences between DA and NDA patients in illness behaviour variables. DA patients, compared to NDA patients, shared more syringes, contracted more sexually transmitted diseases, believed HIV was transmitted to them through blood, were conscious of having practised risky behaviour and took the diagnosis test for this reason, regarded their disease as a more serious one, had worse expectations about the effectiveness of treatment and had a worse relationship with the physician. Moreover, the tendency observed is that DA patients have a more negative pattern of adherence to treatment compared with NDA patients, although the differences are not statistically significant.

Key words: HIV, AIDS, adherence to treatment, substance abuse.

\section{INTRODUCCIÓN}

A pesar de que el tratamiento farmacológico contra la infección por VIH a través de

\footnotetext{
Correspondencia: Rafael Ballester, Dpto. de Psicología Básica, Clínica y Psicobiología, Facultad de Ciencias Humanas y Sociales, Universitat Jaume I de Castello, 12080 Castellón, España. Correo-e: rballest@psb.uji.es Agradecimientos: Este trabajo ha sido posible en parte gracias a las ayudas para la realización de proyectos de investigación concedidas por el Plan del SIDA de la Direcció General de Salut Pública de la Generalitat Valenciana (1/2000) y por la Universitat Jaume I de Castello-Fundació Bancaixa (P1.1 A 2000-05).
}

Inhibidores de la Transcriptasa Inversa y de la Proteasa es hoy más efectivo que nunca, son cada vez más los clínicos que denuncian los bajos niveles de adhesión al tratamiento que presentan estos pacientes (Aversa y Kimberlin, 1996; Ballester, Reinoso, García y Campos, 2000; Chesney, 1997; Gwadz et al., 1999; Ickovics y Meisler, 1997; Kalichman, 1998; López et al., 1999; Samet et al., 1992; Torres et al., 1999).

De esta situación se deriva la importancia de conocer los factores relaciona- 
dos con los problemas de adhesión al tratamiento (Kelly y Kalichman, 2002), con el fin de tenerlos en cuenta a la hora de implementar programas que tengan como objetivo mejorar esta adhesion (en el trabajo de Ballester, 2002, se puede encontrar una revisión teórica al respecto). Entre otros aspectos conocemos la relevancia que pueden tener variables como las creencias culturales de los pacientes (Erwin y Peters, 1999), la percepción acerca de la eficacia del tratamiento (Mehta, Moore y Graham, 1997), la complejidad del mismo (Catz, Kelly, Bogart, Benostch y McAuliffe, 2000; Murphy, Johnston y Martin, 2000), la intolerancia a los efectos secundarios (Rabkin y Chesney, 1998; Roca, Gómez y Arnedo, 2000), la creencia acerca de la toxicidad de los fármacos (Smith et al.,1997), la relación con el facultativo (Gerbert, Love y Caspers, 1999; Roberts, 2000), la inestabilidad en las vidas de los pacientes (Bangsburg, Tulsky, Hecht y Moss, 1997), el ánimo depresivo (Avants, Margolin, Warburton, Hawkins y Shi, 2001; Singh et al., 1996;), la falta de apoyo social (Catz, McClure, Jones y Brantley, 1999; Remor, 2002) o simplemente el olvido (Hecht, 1997).

Algunos autores también han destacado la relevancia que pueden tener las mismas características de los pacientes respecto a sus hábitos de salud, como por ejemplo el abuso de drogas (Gordillo et al., 1999; Gwadz et al., 1999; Haubrich et al., 1999; Kaplan et al., 1999; Moatti et al., 2000; Pedreira, 1999; Román et al., 1999; Singh et al. 1996) como una de las variables más importantes en su efecto negativo sobre la adhesión al tratamiento de los pacientes con infección por VIH.

\section{Objetivo}

El objetivo de este trabajo es analizar la posible existencia de un patrón diferen- cial de conducta de enfermedad y adhesión al tratamiento de la infección por VIH en pacientes que presentan o han presentado un consumo abusivo de drogas. Dentro de lo que consideramos patrón de "adhesión al tratamiento" incluimos variables como la toma de los fármacos, el seguimiento de las visitas médicas y el cambio de hábitos. Pero también consideramos otras variables más generales de conducta de enfermedad que a su vez pueden estar modulando aquéllas, tales como los estresores a los que se enfrenta el paciente, la valoración acerca de la gravedad de la enfermedad, las expectativas respecto al tratamiento o la relación con el facultativo.

\section{Muestra}

La muestra utilizada se compone de 70 personas con infección por VIH/SIDA, la mayor parte de los cuales son pacientes del Servicio de Medicina Interna del Hospital General de Castellón. De los 70 pacientes, 38 presentan una historia de abuso de drogas (AD) y 32 no la presen$\tan (\mathrm{NAD})$. Por sexos, 43 sujetos $(61 \%)$ son hombres y 27 (39\%) son mujeres. La media de edad es de 33 (entre 23 y 62 años) (DT= 8,6). Por lo que respecta al estado civil, el 45 son solteros/as, el $21 \%$ son casados/as, el $22 \%$ son separados/as y el $12 \%$ son viudos/as. Finalmente, en cuanto a la duración de la enfermedad la media de años desde su conocimiento se sitúa en 7,5 años (DT $=4,3$ ) (entre 6 meses y 15 años).

\section{Instrumentos y procedimiento}

Se utilizó una entrevista estructurada (Entrevista de Adhesión al Tratamiento, EAT-VIH, de Ballester, Reinoso, García y Campos, 2000), compuesta por 29 preguntas de respuesta abierta en algunos 
casos y cerrada (elección múltiple) en otros. Aunque los indicadores de adhesión al tratamiento utilizados en este trabajo se basan en el autoinforme del sujeto, algunos trabajos demuestran que el autoinforme de los pacientes a este respecto constituye un buen predictor de la eficacia del tratamiento farmacológico (Haubrich et al, 1999). La EAT-VIH fue administrada de modo individual, voluntario y tras haber obtenido el consentimiento informado de los pacientes.

\section{Análisis estadísticos}

Con el fin de poder analizar las posibles diferencias en las distintas variables evaluadas en función de la historia de consumo de drogas de los pacientes se ha utilizado la prueba Chi cuadrado del programa estadístico SPSS-PC para las variables de respuesta categórica (la mayoria) y la prueba $t$ de Student únicamente para analizar las diferencias en la variable edad, duración de la enfermedad y valoración de la gravedad de la enfermedad.

\section{Resultados}

En primer lugar, hay que decir que no encontramos diferencias significativas entre los grupos de pacientes con/sin historia de abuso de drogas (AD/NAD) en cuanto al sexo, siendo hombres el $55 \%$ de los NAD frente al $\mathbf{7 0} \%$ de los AD. Tampoco encontramos diferencias significativas en la edad de ambos grupos ni en la duración de su enfermedad. Así, la edad media de los NAD es de 35,7 (DT = 11,4) y la de los AD es de 34,9 (DT =6,3) $(t=1,16, p<0,21)$. En cuanto a la duración de su enfermedad, la media de los NAD es de 6,6 años (DT $=3,6)$ y la de los $\mathrm{AD}$ es de 7,4 años (DT $=4,6)(t=-1,10, p$ $<0,23)$. Se trata, pues, de dos grupos de pacientes estadísticamente similares en estas variables. A continuación, expondremos los principales resultados en distintos bloques en función del contenido de las variables evaluadas.

Respecto al primer bloque de resultados, relacionado con los comportamientos y factores de riesgo, como se puede apreciar en la Tabla 1, los pacientes con historia de abuso de drogas (AD) presentan

Tabla 1. Diferencias en comportamientos y factores de riesgo

\begin{tabular}{|c|c|c|c|c|}
\hline Variables & & $\begin{array}{c}\text { NAD } \\
(\mathrm{N}=32)\end{array}$ & $\begin{array}{c}A D \\
(N=38)\end{array}$ & $\begin{array}{l}\text { Chi } \\
\text { cuadrado }\end{array}$ \\
\hline \multirow[t]{6}{*}{ Comportamientos de riesgo } & Ha compartido jeringas & 5,6 & 90,0 & $43,12 * \star *$ \\
\hline & $\begin{array}{l}\text { Ha tenido contactos sexuales con } \\
\text { seropositivos homosex o bisex }\end{array}$ & 38,9 & 46,0 & 0,27 \\
\hline & Rel. sex. Sin preservativo & $\mathbf{3 3}, \mathbf{3}$ & 48,0 & 1,15 \\
\hline & Ha ejercido la prostitución & 0 & 12,0 & 2,37 \\
\hline & $\begin{array}{l}\text { Ha mantenido contactos sexuales } \\
\text { con prostitutas/os }\end{array}$ & 27,8 & 42,0 & 1,13 \\
\hline & $\begin{array}{l}\text { Ha tenido enfermedades de trans- } \\
\text { misión sexual, hepatitis B o C }\end{array}$ & 22,2 & 76,0 & $17,75^{* * *}$ \\
\hline \multirow[t]{3}{*}{ Vía percibida de infección } & Sexual & 75,0 & 12,0 & $32,38 * \star \star$ \\
\hline & Jeringas & 6,3 & 74,0 & \\
\hline & No sabe & 12,5 & 6,0 & \\
\hline Cambio de hábitos & Sí & 75,0 & 89,6 & 2,11 \\
\hline
\end{tabular}

Nota: NAD: Pacientes sin historia de abuso de drogas; AD: Pacientes con historia de abuso de drogas. ${ }^{\star \star *} p<0,001$ 
mayores porcentajes absolutamente en todos los factores de riesgo: tienen más contactos sexuales con seropositivos homosexuales o bisexuales, mantienen más relaciones esporádicas sin usar preservativo, ejercen más la prostitución y tienen más relaciones con trabajadores sexuales. Sin embargo, únicamente dos de las diferencias entre ambos grupos resultan estadísticamente significativas, concretamente el compartir jeringas y el haber padecido enfermedades de transmisión sexual, hepatitis B o C. En cuanto a la vía por la que los pacientes creían haberse infectado, lógicamente encontramos diferencias significativas entre ambos grupos. La vía percibida de infección era la sexual para la mayoría de los NAD y la sanguínea (intercambio de jeringas) para la mayoría de los AD. Finalmente, dentro de este primer bloque relacionado con los comportamientos de riesgo, encontramos un mayor porcentaje de cambio de hábitos en los $A D$ que en los NAD que, no obstante, no llega a producir diferencias estadísticamente significativas. De todos modos, estos resultados podrían estar sesgados porque algunos de los sujetos de la muestra procedían precisamente de un programa de deshabituación a las drogas.
En el segundo bloque, relacionado con el diagnóstico de la infección, como se observa en la Tabla 2, encontramos diferencias significativas en la importancia de encontrarse mal (chi cuadrado $=6,05$, $p<0,01$ ) y ser consciente de las prácticas de riesgo (chi cuadrado $=8,81, p<0,00$ ) como motivos para que los sujetos con/sin historia de abuso de drogas acudan a hacerse las pruebas diagnósticas, siendo la primera más importante en los NAD y la segunda en los AD. Por lo demás, aún sin llegar a alcanzar significación estadística, tras el diagnóstico, los NAD se enfrentan en mayor medida a problemas de pareja y los AD a problemas con la familia, amigos o trabajo, siendo el malestar emocional algo más frecuente en los primeros que en los segundos. Asimismo los pacientes AD perciben menor apoyo social tras el diagnóstico.

El tercer bloque de resultados guarda relación con las creencias que los pacientes tienen acerca de su enfermedad, tanto por lo que se refiere a su gravedad como a su duración. En la Tabla 3 se puede apreciar cómo los pacientes $\mathrm{AD}$ valoran su enfermedad como más grave que los NAD, alcanzando las diferencias sig-

Tabla 2. Diferencias en variables relacionadas con el diagnóstico

\begin{tabular}{llcrc}
\hline Variables & & $\begin{array}{c}\text { NAD } \\
(\mathrm{N}=32)\end{array}$ & $\begin{array}{c}\text { AD } \\
(\mathrm{N}=38)\end{array}$ & $\begin{array}{c}\text { Chi } \\
\text { cuadrado }\end{array}$ \\
\hline $\begin{array}{l}\text { Motivo para realizarse las } \\
\text { pruebas diagnósticas }\end{array}$ & Encontrarse mal & 27,8 & 6,0 & $6,05^{\star *}$ \\
& Por recomendación & 22,2 & 26,0 & 0,10 \\
& Curiosidad & 5,6 & 4,0 & 0,07 \\
& Conciencia de prácticas de riesgo & 0 & 36,0 & $8,81{ }^{\star *}$ \\
& Chequeo rutinario & 27,8 & 18,0 & 0,77 \\
\hline Problemas tras el diagnóstico & Problemas con familia & 5,6 & 16,3 & 1,31 \\
de la infección & Ruptura con pareja & 16,7 & 6,1 & 1,79 \\
& Malestar emoc. o depres. & 77,8 & 71,4 & 0,27 \\
& Problemas en el trabajo & 5,6 & 8,2 & 0,13 \\
\hline Percepción apoyo social & Problemas con los amigos & 0 & 6,1 & 1,15 \\
\hline
\end{tabular}

Nota: NAD: Pacientes sin historia de abuso de drogas; AD: Pacientes con historia de abuso de drogas. * " $p<0,01$ 
Tabla 3. Diferencias en creencias acerca de la enfermedad

\begin{tabular}{llccc}
\hline Variables & & $\begin{array}{c}\mathrm{NAD} \\
(\mathrm{N}=32)\end{array}$ & $\begin{array}{c}\mathrm{AD} \\
(\mathrm{N}=38)\end{array}$ & $\begin{array}{c}\text { Chi } \\
\text { cuadrado }\end{array}$ \\
\hline Valoración de la gravedad & Leve & 11,1 & 10,0 & $10,65^{*}$ \\
& Moderada & 33,3 & 22,0 & \\
& Grave & 11,1 & 50,0 & \\
& Muy grave & 44,4 & 16,0 & \\
\hline Creencia duración de la & Nunca se curará & 28,6 & 27,9 & 2,65 \\
enfermedad & Tardará & 14,3 & 20,9 & \\
& Durac. Normal & 0 & 2,3 & \\
& Pronto se curará & 35,7 & 18,6 & \\
\hline
\end{tabular}

Nota: NAD: Pacientes sin historia de abuso de drogas; AD: Pacientes con historia de abuso de drogas. ${ }^{*} p<0,05$

nificación estadística (chi cuadrado $=$ $10,65, p<0,03$ ). Sin embargo, al comparar las medias de ambos grupos y aplicar la prueba $t$ de Student, los resultados no nos muestran diferencias significativas, siendo la media de los NAD de 2,9 (DT = $1,1)$ y la de los AD de 2,7 (DT = 0,9). La misma tendencia es hallada en la creencia de ambos grupos respecto a la duración de la enfermedad, que es más optimista en los NAD que en los $A D$, aunque aquí las diferencias no son estadísticamente significativas.

El cuarto bloque de resultados se refiere al tratamiento farmacológico de los pacientes. Como se puede ver en la Tabla 4, aún sin alcanzar significación estadística, encontramos un mayor porcentaje de pacientes sin historia de abuso de drogas que toman un tratamiento, aunque los pacientes con historia de abuso de drogas que siguen un tratamiento lo valoran más positivamente. Encontramos también unas expectativas más optimistas en los NAD respecto a las consecuencias de seguir un tratamiento y en este ámbito sí encontramos diferencias estadísticamente significativas (chi cuadrado $=3,56, p<0,05)$. Coherentemente, las expectativas de los NAD respecto a las consecuencias de no tomar un tratamiento son más negativas que las de los $\mathrm{AD}$.

El quinto bloque de resultados se refiere a los problemas de adhesión al tratamiento farmacológico. Cono se observa en la Tabla 5, podemos decir que en

Tabla 4. Diferencias en variables relacionadas con el tratamiento farmacológico

\begin{tabular}{llccc}
\hline Variables & & $\begin{array}{c}\mathrm{NAD} \\
(\mathrm{N}=32)\end{array}$ & $\begin{array}{c}\mathrm{AD} \\
(\mathrm{N}=38)\end{array}$ & $\begin{array}{c}\text { Chi } \\
\text { cuadrado }\end{array}$ \\
\hline $\begin{array}{l}\text { Valoración acerca de la } \\
\text { adecuación del tratamiento }\end{array}$ & Valoración positiva & 76,9 & $\mathbf{8 2 , 9}$ & 3,21 \\
\hline Expectativas Tratamiento & Curación & 31,3 & 15,2 & 1,95 \\
& Encontrarse mejor & 37,5 & 15,2 & 3,56 \\
& Disminuir el avance de enferm & 68,8 & 73,9 & 0,16 \\
& No se espera nada & 0 & 4,3 & 0,72 \\
\hline Expectativa sin tratamiento & Agravamiento de enfermedad & 87,5 & 67,4 & 2,41 \\
& Nunca habría curación & 6,3 & 13,0 & 0,55 \\
& Muerte & 31,3 & 15,2 & 1,95 \\
\hline
\end{tabular}

Nota: NAD: Pacientes sin historia de abuso de drogas; AD: Pacientes con historia de abuso de drogas. ${ }^{*} p<0,05$ 
Tabla 5. Diferencias en problemas de adhesión al tratamiento farmacológico

\begin{tabular}{llccc}
\hline Variables & & $\begin{array}{c}\mathrm{NAD} \\
(\mathrm{N}=32)\end{array}$ & $\begin{array}{c}\mathrm{AD} \\
(\mathrm{N}=38)\end{array}$ & $\begin{array}{c}\text { Chi } \\
\text { cuadrado }\end{array}$ \\
\hline $\begin{array}{l}\text { Dejar de tomar el tratamiento } \\
\text { alguna vez }\end{array}$ & $\mathrm{Sí}$ & 37,5 & 60,0 & 2,41 \\
\hline Causas de no tomar fármacos & Número excesivo de pastillas & 0 & 26,7 & 2,06 \\
& $\begin{array}{l}\text { Efectos secundarios } \\
\text { Olvido }\end{array}$ & 20,0 & 23,3 & 0,03 \\
& $\begin{array}{l}\text { Creencia de que el tratamiento no } \\
\text { hace ningún efecto }\end{array}$ & 80,0 & 36,7 & 3,29 \\
& & 0 & 3,3 & 0,17 \\
\hline $\begin{array}{l}\text { Preocupación por efectos } \\
\text { concretos de medicación }\end{array}$ & Sí & 36,4 & 57,5 & 1,55 \\
\hline
\end{tabular}

Nota: NAD: Pacientes sin historia de abuso de drogas; AD: Pacientes con historia de abuso de drogas.

general los pacientes con historia de abuso de drogas comparados con los pacientes sin historia de abuso de drogas, dejan de tomar los fármacos con mayor frecuencia, se encuentran más preocupados por los efectos secundarios concretos de la medicación y en su abandono del tratamiento pesa más el número excesivo de pastillas frente al olvido que resulta más importante en los pacientes sin historia de abuso de drogas. Sin embargo, no detectamos diferencias estadísticamente significativas en ninguna de estas variables entre ambos grupos.

El sexto bloque de resultados también se encuentra estrechamente vinculado con la adhesión de los pacientes, al explorar la conducta de acudir a las visitas cada vez que el médico cita al pacien- te y las causas en el caso de que no lo hagan. En la Tabla 6 se aprecia cómo dentro de este bloque tampoco encontramos diferencias significativas estadísticamente, aunque los pacientes con historia de abuso de drogas parecen dejar de acudir con mayor frecuencia a las revisiones médicas y en general, se muestran menos preocupados que los pacientes sin historia de abuso de drogas ante las mismas.

Finalmente, el séptimo bloque de resultados se ocupa de los datos referidos a la relación entre el facultativo y el paciente, aspecto que algunos autores han destacado como potencialmente relevante en la adhesión al tratamiento. Como se puede ver en la Tabla 7 , en prácticamente todas las variables los pacientes NAD muestran porcentajes más altos

Tabla 6. Diferencias en el seguimiento de las visitas médicas

\begin{tabular}{llccc}
\hline Variables & & $\begin{array}{c}\mathrm{NAD} \\
(\mathrm{N}=32)\end{array}$ & $\begin{array}{c}\mathrm{AD} \\
(\mathrm{N}=38)\end{array}$ & $\begin{array}{c}\text { Chi } \\
\text { cuadrado }\end{array}$ \\
\hline Acudir a las revisiones & $\mathrm{Si}$ & 100 & $\mathbf{8 5 , 7}$ & 2,72 \\
\hline $\begin{array}{l}\text { Causas de no acudir a las } \\
\text { revisiones médicas }\end{array}$ & $\begin{array}{l}\text { Preocupación por saber que la } \\
\text { enfermedad no responde al } \\
\text { tratamiento }\end{array}$ & & & \\
& $\begin{array}{l}\text { Idem haber empeorado } \\
\text { Excesiva frecuencia de las visitas }\end{array}$ & $\mathbf{3 8 , 5}$ & 22,2 & 1,39 \\
& Mala relación con el médico & 0 & 51,1 & 0,03 \\
& No le preocupa nada & 7,7 & 4,4 & 0,22 \\
& & 24,4 & 0,59 \\
\hline
\end{tabular}

Nota: NAD: Pacientes sin historia de abuso de drogas; AD: Pacientes con historia de abuso de drogas. 
Tabla 7. Diferencias en la relación médico-paciente

\begin{tabular}{lcccc}
\hline Variables & & $\begin{array}{c}\mathrm{NAD} \\
(\mathrm{N}=32)\end{array}$ & $\begin{array}{c}\mathrm{AD} \\
(\mathrm{N}=38)\end{array}$ & $\begin{array}{c}\text { Chi } \\
\text { cuadrado }\end{array}$ \\
\hline Relación con el médico & $\begin{array}{c}\text { Comunicación al médico de preo- } \\
\text { cupación por efectos secundarios }\end{array}$ & 75,0 & 79,2 & 0,03 \\
& $\begin{array}{c}\text { Preguntar dudas al médico acerca } \\
\text { de la enfermedad y tratamiento }\end{array}$ & 100 & 75,7 & $3,57 *$ \\
& $\begin{array}{l}\text { Se siente comprendido por médico } \\
\text { Buena relación con el médico }\end{array}$ & 93,8 & 75,5 & 3,89 \\
& $\begin{array}{l}\text { El médico le ha informado sobre la } \\
\text { enfermedad }\end{array}$ & 100 & 83,3 & 1,51 \\
& El médico le dedica suficiente tiempo & 88,2 & 72,9 & 2,04 \\
\hline
\end{tabular}

Nota: NAD: Pacientes sin historia de abuso de drogas; AD: Pacientes con historia de abuso de drogas. ${ }^{\star} p<0,05$

que los pacientes AD. Únicamente los porcentajes de respuesta son muy aproximados e incluso superiores en los $\mathrm{AD}$ ante la pregunta de si han comunicado al médico sus preocupaciones acerca de los efectos secundarios de los fármacos. Por tanto, podemos decir que, aunque únicamente se obtiene significación estadística en una de las variables comparadas, la de preguntar las dudas al facultativo (chi cuadrado $=3,57, p<0,05$ ), en general, los pacientes con historia de abuso de drogas presentan una peor relación con el mismo, expresada a través de una peor comunicación, menor confianza para preguntarle sus dudas, menor sensación de que éste les comprende y menor satisfacción con el tiempo que se les dedica.

\section{CONCLUSIONES Y DISCUSIÓN}

El análisis global de los resultados expuestos anteriormente nos permite decir que existen algunas diferencias entre los pacientes seropositivos con y sin historia de abuso de drogas en su conducta de enfermedad pero en el caso de las variables directamente relacionadas con la adhesión al tratamiento estas diferencias son menores, no alcanzando en ningún caso la significación estadística. De entre un total de 49 variables com- paradas entre ambos grupos, hemos obtenido diferencias significativas en tan sólo 8 de ellas, esto es, en el $16,3 \%$ de las variables.

De éstas, tres se encuentran en el bloque de comportamientos/factores de riesgo que, como resulta lógico, difieren mucho en función de la historia de abuso de drogas de los pacientes. Así, los pacientes $A D$ han compartido jeringas en mayor medida, han padecido más enfermedades de transmisión sexual, hepatitis $B$ o $C$, y su vía percibida de infección es la sanguínea a través de la conducta de compartir jeringas. También hemos encontrado dos diferencias significativas en los motivos que llevan a los pacientes a realizarse las pruebas diagnósticas. En este sentido, los pacientes NAD suelen, en mucha mayor medida que los $A D$, realizarse las pruebas por encontrarse mal, mientras que lo contrario ocurre respecto a la conciencia de haber realizado prácticas de riesgo, que es mucho mayor como factor motivador de realizarse las pruebas en éstos últimos. Hallamos la quinta diferencia significativa en la valoración de la gravedad de la enfermedad que parece ser mayor en los pacientes AD. La sexta diferencia se encuentra en la expectativa que presentan respecto a los resultados del tratamiento. Los pacientes NAD esperan en mucha mayor 
medida que el otro grupo "encontrarse mejor" con el tratamiento. Y finalmente, la última diferencia significativa se da en una de las variables referidas a la relación con el médico. Los pacientes NAD preguntan en mayor medida al médico sus dudas acerca de la enfermedad y su tratamiento.

Al margen de las variables en las que hemos encontrado diferencias significativas estadísticamente, apreciamos en nuestro estudio ciertas tendencias cuyo análisis puede ser clínicamente interesante y por ello las hemos comentado en el apartado de resultados, pero que de ningún modo apoyan la idea de que la historia de consumo de drogas constituya una variable con un importante papel modulador en la adhesión al tratamiento de los pacientes con infección por VIH/SIDA.

En la literatura científica al respecto, podemos encontrar un buen número de trabajos científicos que señalan al abuso de drogas como una de las variables más relevantes en su efecto modulador no sólo sobre la adhesión al tratamiento de los pacientes con infección por VIH, sino también sobre la probabilidad de infección a través de las conductas de riesgo. Un ejemplo de este segundo aspecto es el trabajo de Anderson et al. (1999) en el que se evidencia que los consumidores de drogas también tienen más probabilidad de llevar a cabo otras conductas sexuales de riesgo, incrementando así la probabilidad de infección. McBride, Weatherby, Inciardi y Gillespie (1999) en su estudio hablan incluso de una relación significativa entre la frecuencia de ese consumo de drogas y la vulnerabilidad percibida a la infección por VIH. Finalmente, en la investigación llevada a cabo por Deas, Brady, White y Campbell (1999), en la que se compara adolescentes consumidores de drogas con otros que no lo son, se encontró que en los primeros se daba un desfase significativamen- te mayor entre conocimiento y conducta por lo que respecta a la prevención del SIDA, esto es, los adolescentes consumidores de drogas realizaban también más conductas sexuales de riesgo. Los autores apelan a la impulsividad asociada al consumo de drogas como posible factor explicativo de la mayor frecuencia de conductas sexuales de riesgo.

Si la relación entre consumo de drogas y riesgo ante la infección del VIH parece bastante clara, no lo es tanto, la relación entre este consumo y la adhesión al tratamiento, una vez la persona ya está afectada por esta enfermedad.

Algunos estudios encuentran evidencias a favor de la relación entre el consumo de alcohol o de drogas en general y la adhesión al tratamiento. Es el caso del trabajo de Petry (1999) en el que se observa que el consumo excesivo de alcohol suele estar asociado a una menor adhesión al tratamiento del VIH. También Gwadz et al. (1999), tras evaluar a una muestra de 210 pacientes, pudieron comprobar que los que se abstenían de consumir drogas tenían mayor probabilidad de adherirse al tratamiento. Incluso, algunos autores como Sorensen et al. (1998) indican que entre las intervenciones para mejorar la adhesión al tratamiento deberían desarrollarse estrategias específicas para los pacientes con un consumo abusivo de drogas.

Otros estudios matizan algo más y no hablan de relación entre adhesión al tratamiento y consumo o abuso de drogas en general, sino de relación entre la adhesión y el uso de drogas intravenosas. Por ejemplo, Gordillo et al., (1999) en su estudio llevado a cabo con 366 pacientes encontraron que presentaban peor adhesión los jóvenes, los que puntuaban más alto en depresión, los que percibían menor apoyo social y los usuarios de drogas por vía intravenosa. También Kaplan et al., (1999) hallaron resultados similares con su muestra de 148 pacientes: en 
la adhesión no parecía influir el sexo, la edad, ni la etnia, pero sí el hecho de que los pacientes fueran usuarios de drogas inyectadas.

En tercer lugar, habría que hablar de los estudios como el de Haubrich et al., (1999) que diferencian entre consumo de drogas y pertenencia o no a determinados grupos de riesgo. En el trabajo citado, los autores evaluaron a una muestra de 173 pacientes y concluyeron que, en efecto, el uso lúdico de alcohol y otras drogas estaba asociado a una menor adhesión al tratamiento, mientras que otras variables como el estadio de la enfermedad, la edad, sexo, educación o grupo de riesgo no lo estaban. Cuando estos autores utilizan la ya desfasada nomenclatura de "grupo de riesgo", se están refiriendo al hecho de que sean toxićrmanos o drogodependientes, homosexuales o heterosexuales y en el caso de drogodependientes, están significando la existencia de una historia de abuso de drogas independientemente de que en el momento actual se sigan consumiendo o no.

Quizás esta distinción pueda explicar a la vez los resultados de los estudios anteriormente mencionados y otros resultados como los del estudio de Kastrissios et al. (1998) con 41 pacientes seropositivos en el que se concluía que el tipo de medicación tenía una influencia clara sobre la adhesión pero no parecían especialmente relevantes en general las características de los pacientes, como su pertenencia o no a "grupos de riesgo". También es digna de mención la investigación de Holzemer et al., (1999) llevada a cabo con un total de 420 pacientes extraídos de 7 ciudades de los EEUU y de todo tipo de hospitales y servicios de asistencia, privados y públicos. Estos autores encontraron una relación significativa entre adhesion y algunos aspectos como sentirse felices y bien cuidados, dedicar tiempo a las cosas importantes o encontrarle sentido a la vida, pero no entre adhesión y otras variables como la edad, sexo, etnia o la historia de uso de drogas inyectadas.

En nuestro caso, las diferencias que hemos encontrado entre los pacientes con y sin historia de abuso de drogas en relación con la adhesión no pasan de ser meras tendencias que no alcanzan significación estadística, a diferencia de lo que ocurre en otras variables más generales relacionadas con la conducta de enfermedad en las que los pacientes con historia de abuso de drogas sí obtienen un patrón diferencial estadísticamente significativo. La escasez de diferencias significativas obtenidas en nuestro estudio podría ser debida al reducido tamaño de la muestra estudiada (tan sólo 70 pacientes), pero también al hecho de que lo que evaluábamos en nuestros pacientes era la historia de abuso de drogas y no el hecho de que en el momento actual estuvieran consumiéndolas y tuvieran un consumo abusivo de las mismas. Precisamente, algunos de nuestros pacientes provenían de centros donde se estaban aplicando programas de deshabituación. Por todo ello, de nuestro estudio no se puede deducir que el consumo actual de drogas no tenga ninguna influencia sobre la adhesión al tratamiento, sino tan sólo que la historia de ese consumo, es decir, el hecho de que se hayan consumido en el pasado, no tiene por qué tener un efecto negativo sobre la adhesión, lo cual está en la línea de los resultados obtenidos por Kastrissios et al. (1998), Haubrich et al., (1999) y Holzemer et al., (1999) sin por ello contradecir las conclusiones de otros estudios como los de Gwadz et al. (1999), Gordillo et al., (1999) o Kaplan et al., (1999), en los que la relación hallada se daba entre la adhesión y el consumo actual de drogas.

En nuestra opinión, es necesario seguir realizando más estudios intentando, por un lado, utilizar grandes muestras que puedan aclarar las posibles relaciones 
existentes y por otro lado, prestando mucha atención a cuestiones metodologicas que podrían explicar los resultados divergentes entre los distintos estudios realizados hasta el momento, como establecer claramente la distinción entre ser usuario de drogas, lo cual puede suponer un consumo esporádico, presentar un consumo abusivo de drogas, o tener una historia de abuso de drogas, que no habla de qué está ocurriendo en el momento de la evaluación del paciente. Además habría que aclarar el tipo de drogas del que estamos hablando para poder comparar los resultados obtenidos en los diferentes estudios.

\section{REFERENCIAS BIBLIOGRÁFICAS}

Anderson, J., Wilson, R., Barker, P., Doll, L., Jones, T., y Holtgrave, D. (1999). Prevalence of sexual and drug-related HIV risk behaviors in the U.S. adult population: results of the 1996 National Household Survey on Drug Abuse. Journal of Acquired Immune Deficiency Syndrome, 21, 148-156.

Avants, S., Margolin, A., Warburton, L., Hawkins, K., y Shi, J. (2001). Predictors of nonadherence to HIV-related medication regimens during methadone stabilization. American Journal on Addictions, 10, 6978.

Aversa, S.L., y Kimberlin, C. (1996). Psychosocial aspects of antiretroviral medication use among HIV patients. Patient Education and Counselling, 29, 207-219.

Ballester, R. (2002). Adhesión terapéutica: revisión histórica y estado de la cuestión en la Infección por VIH/SIDA. Revista de Psicopatología y Psicología Clínica, 7, 151175.

Ballester, R., Reinoso, I., García, S., y Campos, A. (2000). Adhesión al tratamiento en la infección por VIH. Anólisis y Modificación de Conducta, 109, 689-718.

Bangsburg, D., Tulsky, J.P., Hecht, F.M., y Moss, A.R. (1997). Protease inhibitors in the Homeless. Journal of the American
Medical Association, 278, 63-65.

Catz, S., Kelly, J., Bogart, L., Benotsch, E., y McAuliffe, T. (2000). Patterns, correlates and barriers to medication adherence among persons prescribed new treatments for HIV disease. Health Psychology, 19, 124-133.

Catz, S., McClure, J., Jones, G., y Brantley, P. (1999). Predictors of outpatient medical appointment attendance among persons with HIV. AIDS Care, 11, 361-373.

Chesney, M.A. (1997). New antiretroviral therapies: adherence challenges and strategies. Evolving HIV treatments: Advances and the challenge of adherence, $37^{\text {th }}$ ICA. AC Symposium, Toronto, Canada, September.

Deas, N., Brady, K., White, R., y Campbell, S. (1999). HIV-risk behaviors in adolescent substance abusers. Journal of Substance Abuse Treatment, 16, 169-72.

Erwin, J., y Peters, B. (1999). Treatment issues for HIV+ Africans in London. Social Science and Medicine, 49, 1519-1528.

Gerbert, B., Love, C., y Caspers, N. (1999). Making all the Difference in the World: How physicians can help HIV-seropositive patients become more involved in their health care. AIDS Patient Care and STDS, 13, 29-39.

Gordillo, M.V., Del Amo, J., Soriano, V., y González-Lahoz, J. (1999). Sociodemographic and psychological variables influencing adherence to antiretroviral therapy. AIDS, 13, 1763-1769.

Gwadz, M., De-Vogli, R., Rotheram-Borus, M.J., Diaz, M., Cisek, T., James, N., y Tottenham, N. (1999). Behavioral practices regarding combination therapies for HIV/AIDS. Journal of Sex Education and Therapy, 24, 81-88.

Haubrich, R., Little, S., Currier, J., Forthal, D., Kemper, C., Beall, G., Johnson, D., Dube, M., Hwang, J., y McCutchan, J. (1999). The value of patient-reported adherence to antiretroviral therapy in predicting virologic and immunologic response. AIDS, 13, 1099-1107.

Hecht, F.M. (1997, December). Adherence to HIV treatment. Paper presented at the meeting of Clinical Care of the AIDS Patients, San Francisco, California. 
Holzemer, W., Corless, I., Nokes, K., Turner, J., Brown, M., Powell-Cope, G., Inouye, J., Henry, S., Nicholas, P., y Portillo, C. (1999). Predictors of Self-Reported Adherence in Persons living with HIV-disease. Patient Care and STDS, 13, 185-197.

Ickovics, J., y Meisler, A.W. (1997). Adherence in AIDS clinical trials: a framework for clinical research and clinical care. Journal of Clinical Epidemiology, 50, 385-391.

Kalichman, S.C. (1998). Understanding AIDS: Advances in Research and Treatment. Washington: American Psychological Association.

Kaplan, J., Parham, D., Soto-Torres, L., van Dyck, K., Greaves, J., Rauch, K., Ellis, B., y Amandus, H. (1999). Adherence to guidelines for antiretroviral therapy and for preventing opportunistic infections in HIVinfected adults and adolescents in Ryan White-funded facilities in the United States. Journal of Acquired Immune Deficiency Syndrome, 3, 228-235.

Kastrissios, H., Suárez, J.R., Katzenstein, D., Girard, P., Sheiner, L., y Blaschke, T. (1998). Characterizing patterns of drugtaking behavior with a multiple drug regimen in an AIDS clinical trial. AIDS, 12, 2295-2303.

Kelly, J.A., y Kalichman, S. (2002). Behavioral research in HIV/AIDS primary and secondary prevention: recent advances and future directions. Journal of Consulting and Clinical Psychology, 70, 629-639.

López, A., Juega, J., Baliñas, J., Pita, S., Margusino, L., Picallos, M., y Pedreira, J.D. (1999). Cumplimiento terapéutico en pacientes VIH+. Publicación Oficial de la Sociedad Española Interdisciplinaria del SIDA, 10, 43.

McBride, D., Weatherby, N., Inciardi, J., y Gillespie, S. (1999). AIDS susceptibility in a migrant population and behavior. Substance Use and Misuse, 34, 633-652.

Mehta, S., Moore, R.D., y Graham, N.M.H. (1997). Potential factors affecting adherence with HIV therapy. AIDS, 11, 1665-1670. Moatti, J., Carrieri, M., Spire, B., Gastaut, J., Cassuto, J., y Moreau, J. (2000). Adherence to HAART in French HIV-infected injecting drug users: the contribution of buprenorphine drug maintenance treatment. The
Manif 2000 study group. AIDS, 14, 151155.

Murphy, D., Johnston, K., y Martin, D. (2000). Barriers to Antiretroviral Adherence among HIV-Infected Adults. AIDS Patient Care and STDs, 14, 47-58.

Pedreira, J.D. (1999). Adhesión al tratamiento antirretrovírico del paciente infectado por VIH. Publicación Oficial de la SEISI$D A, 10,167-168$.

Petry, N. (1999). Alcohol use in HIV patients: what we don't know may hurt us. International Journal of STD and AIDS, 10, 561-570.

Rabkin, J.G., y Chesney, M. (1998). Treatment adherence to HIV medications: The Achilles heel of the new therapeutics. En D. Ostrow y S. Kalichman (Eds.), Behavioral and mental health impacts of new HIV therapies. New York: Plenum Press.

Remor, E. (2002). Valoración de la adhesión al tratamiento antirretroviral en pacientes VIH+. Psicothema, 14, 262-267.

Roberts, K.J. (2000). Barriers to and facilitators of HIV-positive patients' adherence to antiretroviral treatment regimens. AIDS Patient Care and STDS, 14, 155-168.

Roca, B., Gómez, C., y Arnedo, A. (2000). A randomized, comparative study of lamivudine plus stavudine, with indinavir or nelfinavir, in treatment-experienced HIVinfected patients. AIDS, 14, 157-161.

Román, M., Nieto, E., Mansilla, J.J., Salas, F. Vallejo M., y García, M.A. (1999). Cumplimiento de la terapia antirretroviral. Publicación Oficial de la SEISIDA, 10, 80.

Samet, J.H., Libman, H., Steger, K.A., Dhwan, R.K., Chen, J., Shevitz, A.H., Dewees-Dunk, R., Leveson, S., Kufe, D., y Caven, D.E. (1992). Compliance with zidovudine therapy in patients infected with HIVtype 1: A cross sectional study in a municipal hospital clinic. American Journal of Medicine, 92, 495-502.

Singh, N., Squier, C., Sivek, C., Wagener, M., Nguyen, M.H., y Yu, V.L. (1996). Determinants of compliance with antiretroviral therapy in patients with human immunodeficiency virus: Prospective assessment with implications for enhancing compliance. AIDS Care, 8, 261-269.

Smith, M., Rapkin, B., Morrison, A., y Kammerman, S. (1997). Zidovudine 
adherence in persons with AIDS. The relation of patient beliefs about medication to self-termination of therapy. Journal of General Internal Medicine, 12, 216-223.

Sorensen, J., Mascovich, A., Wall, T., DePhilippis, D., Batki, S., y Chesney, M. (1998). Medication adherence strategies for drug abusers with HIV/AIDS. AIDS Care, 10, 297-312.

Torres, F.I, Carmona, G., López-Briz, E., Abril, V., Ortega, E., y López, A. (1999). Estudio de adhesión al tratamiento antirretroviral en el Hospital General Universitario de Valencia. Publicación Oficial de la Sociedad Española Interdisciplinaria del SIDA, 10, supl.1. 